\title{
PROPUESTA DE EVALUACIÓN DE PERCEPTIÓN VISUAL COMO RUTINA EN EL EXAMEN DE NIÑOS CON ALTERACIONES GENÉTICAS O NEUROLÓGICAS
}

PROPOSAL OF A VISUAL PERCEPTION OF ASSESSMENT AS ROUTINE IN CHILDREN WITH GENETIC OR NEUROLOGICAL DISORDERS

\section{Nathalya Alessandra Lima Santos ${ }^{1}$, Leslye Camila de Carvalho Silva ${ }^{2}$, Richelle Nogueira Alves Costa ${ }^{3}$,Daniela de Souza Cavalcante, ${ }^{4}$ Raquel Costa Albuquerque. ${ }^{5}$}

\section{RESUMEN}

Se trata de un estudio descriptivo y de observación para verificar y describir la importancia de la utilización de un instrumento de triaje del comportamiento visomotor de niños con alteración genética y/o neurológica. Para recolectar información se elaboró un cuestionario aplicándose la Guía de Examen Visual para análisis del comportamiento visomotor en los niños. Participaron del estudio 22 niños, de los cuales el 81,8\% presentaba Síndrome de Down. De las diez pruebas de que consta la Guía, dos (sonrisa al contacto social y extender el brazo en dirección al objeto visualizado), no fueron respondidas por el 63,6\% de los bebés. A pesar de que la población con Síndrome de Down es más sensible a las alteraciones visuales, fue posible constatar que los niños examinados tuvieron un percentil elevado de respuestas positivas, pudiendo asociarse a la estimulación que reciben. El Terapeuta Ocupacional es el profesional habilitado para trabajar en el desarrollo infantil, debiendo estar atento al desarrollo del sistema visual. Como la guía de examen visual nos da respuestas importantes sobre el comportamiento visual de los niños, además de ser capaz de detectar posibles déficits visuales, quedó demostrada la necesidad de su utilización en los servicios de salud por terapeutas capacitados.

\section{Palabras clave:}

Desarrollo infantil, Percepción visual, Triaje, Terapia Ocupacional.

\footnotetext{
ABSTRACT

1 Terapeuta Ocupacional - Universidade Federal de Pernambuco - UFPE. Endereço: Rua Pero Vaz de Caminha, 95, Ibura, Recife, Pernambuco, Brasil. Telefone: (+55 81) 8769-0286. nathalya.als@hotmail.com

2 Terapeuta Ocupacional - Universidade Federal de Pernambuco - UFPE. leslyecarvalho@hotmail.com

3 Terapia Ocupacional - Universidade Federal de Pernambuco - UFPE. richellenogueira@hotmail.com

4 Terapeuta Ocupacional. Terapia Ocupacional (Universidade Federal de Pernambuco - UFPE). danisouzac@hotmail.com

5 Terapeuta Ocupacional. Doutora em Saúde Materno Infantil (Instituto de Medicina Integral Professor Fernando Figueira - IMIP). Terapia Ocupacional (Universidade Federal de Pernambuco - UFPE). raquel.albuquerque@terra.com.br
} 
This is a descriptive, observational study that aims to determine and describe the importance of using a visuomotor behavior screening tool for children with genetic and / or neurological changes. To collect the data, a questionnaire was designed and the visual assessment script was implemented to analyze the visuomotor behavior of children. The participants were 22 children, where $81.8 \%$ of those with Down syndrome. From the ten tests composing the script, two of them (smile social contact and reach out toward the viewed object), were not answered by $63.3 \%$ of the assessed babies. Despite the population with Down syndrome be easily affected by visual changes, it was found that the children evaluated had high percentile of positive responses, which may be associated with the stimulation that these children receive. The Occupational Therapist is a qualified professional to work with child development and should be alert to the development of the visual system. Since the visual assessment script gives us important answers about the visual behavior of children, as well as being able to detect possible visual deficits, it become evident the need for its use in health services by trained occupational therapists for its application.

\section{Keywords:}

Child development, visual perception, Screening, Occupational Therapy.

\section{INTRODUCCIÓN}

La salud ocular es un importante componente para el desarrollo del niño. Según Ferreira et al (2011), problemas oftalmológicos son capaces de provocar alteraciones en el desarrollo global del niño, toda vez que la visión interfiere en el desarrollo motor y en los aspectos cognitivo y socioemocional.

En los bebés en general, la dificultad para establecer el contacto ocular con la madre o cuidador, así como la falta de interés por objetos y la limitación en la habilidad de fijar y seguir visualmente un objeto en movimiento pueden ser indicios de comprometimiento visual. Otro aspecto observado inmediatamente después de los primeros meses de vida es la dificultad para llevar las manos hasta la línea media o dirigir la mano hacia un objeto presentado en la línea media (Gagliardo, 2003).

Alteraciones en la percepción visual y lentitud en el procesamiento de las informaciones aferentes coexisten con cuadros de desatención, desmotivación y de retraso en la adquisición de importantes marcos en el desarrollo (Pueschel, 2007).

Las alteraciones visuales en niños pueden provenir de diversas etiologías como hidrocefalia, malformaciones cerebrales, hipoxia perinatal, parto prematuro y meningitis, entre otras. Así, es muy común ver pacientes con graves anormalidades neurológicas y oftalmológicas asociadas. La Ilamada deficiencia visual cortical también se ha asociado típicamente a déficits neurológicos, que incluyen la parálisis cerebral, el retraso mental y la hemiparesia (Khetpal \& Donahue, 2007).

Estudios relatan que alteraciones genéticas como el Síndrome de Down se presentan algunas características oculares como ojos con pliegues palpebrales oblicuos y mayor distanciamiento entre ellos, que pueden contribuir a alteraciones en la función visual. Las alteraciones oculares en portadores del síndrome pueden presentar anomalías de grado moderado a severo, provocando en la misma intensidad perjuicios funcionales (Schwartzman, 2003).

En este contexto, si las alteraciones visuales son detectadas en el momento oportuno por medio de la evaluación del comportamiento visual, los niños tendrán la oportunidad de participar en programas de habilitación infantil. El examen utilizado como rutina, principalmente en niños considerados de riesgo, puede ser una medida preventiva, ya que durante el primer año de vida se produce un gran desarrollo visual en el lactante (Gagliardo, Nobre, 2001; Ruas et al., 2006).

Por lo tanto, para la evaluación del comportamiento visual, la detección precoz de alteraciones visuales y las consiguientes estrategias direccionadas a la promoción de la salud ocular se hace evidente la necesidad de un instrumento de triaje rutinario, que permita una evaluación visual precoz para todos los niños (Sperandio, 1999; Aguiar, Cardoso \& Lúcio, 2007).

Según Aragão et al (2013), la evaluación de la función visual permite la detección de posibles alteraciones de la visión para que se inicie la intervención precoz. Junto a ello, los autores afirman que el terapeuta ocupacional es capaz de minimizar los efectos nocivos de tales alteraciones visuales y lo que estas puedan representar para el desarrollo neuropsicomotor.

Tomando como base lo anteriormente expuesto, y ante la importancia del primer año de vida para el desarrollo visual del niño y la necesidad de la utilización del test de triaje visual (Gagliardo, Gonçalves \& Lima, 
2004) para la prevención de daños a la salud en las intervenciones infantiles, este estudio tiene como objetivo evaluar el comportamiento visomotor de niños de la franja etaria comprendida entre los 15 días y los 12 meses, utilizando una guía de triaje visual. Se pretende, así, identificar posibles alteraciones en la función visual, buscando la posibilidad de hacer de esta guía una rutina en la asistencia terapéutica ocupacional de niños en Recife - Brasil, que sufren de síndrome de Down o disfunción neurológica .

\section{MetodoloGía}

Se trata de un estudio descriptivo y observacional, realizado en el período de noviembre a diciembre de 2014, en la Asociación Novo Rumo. Tuvo como población foco niños de una franja etaria comprendida entre los 15 días y los 12 meses, que presentaban alteraciones genéticas o neurológicas.

La Asociación Novo Rumo está localizada en la ciudad de Recife, Pernambuco, Brasil. Se trata de una entidad sin fines lucrativos que ofrece desde 2006 servicios de rehabilitación para niños de 0 a 10 años con síndromes, parálisis cerebral y con deficiencia intelectual o auditiva. Los niños son admitidos por demanda espontánea o encaminados por profesionales de la salud. Entre las posibilidades de asistencia a niños asistidos por la Asociación, se encuentran: Síndrome de Down; Síndrome de Cornelia de Lange; Síndrome de Turner; Malformaciones Congénitas; Trastorno del Espectro Autista; Niños Psicóticos y con Trastorno de Déficit de Atención y/o Hiperactividad, siendo referencia en la atención a bebés con Síndrome de Down (90\% del público).

Estos niños son examinados por los profesionales que componen el equipo, $y$, una vez caracterizada la necesidad de intervención, son acompañados en las terapias adecuadas a sus necesidades. La asistencia se presta por parte de los profesionales de Fisioterapia, Logopedia, Medicina y Terapia Ocupacional.

Los niños que compusieron la muestra del presente estudio fueron conducidos al equipo de investigación para ser examinados por parte del equipo profesional de la Asociación Novo Rumo. Es importante resaltar que independientemente de que estos niños hayan presentado o no alteración en el comportamiento visomotor, fueron examinadas y acompañadas por los demás integrantes del equipo de rehabilitación.
Para la recolección de los datos, se realizó una anamnesis elaborada por la investigadora principal para la identificación del paciente y su familia y el conocimiento de las condiciones clínicas del niño.

Para la evaluación del comportamiento visomotor se empleó la Guía de Triaje para Examen de la Conducta Visual (Gagliardo, Gonçalves \& Lima, 2004) (Cuadro 1), compuesto por diez pruebas que evalúan y cualifican el comportamiento visomotor (funciones óculomotoras y motoras apendiculares), en los primeros tres meses de vida. Fue elaborado a partir de la adaptación de pruebas de la Guía del Examen Neurológico del Recién Nacido (Dargassies, 1977) y de las Escalas Bayley de Desarrollo Infantil (Bayley, 1993). Utiliza como instrumento de evaluación un aro rojo de 12 centímetros de diámetro, suspenso por un cordón, y la cara del examinador, pudiendo cada prueba repetirse hasta tres veces.

Las diez pruebas son: fijación visual, contacto ocular con el examinador, sonrisa como respuesta al contacto social, seguimiento visual horizontal, seguimiento visual vertical, exploración visual del ambiente, exploración visual de la mano, aumento del movimiento de los miembros superiores al visualizar el objeto, extender el brazo en la dirección del objeto visualizado, y, por último, la observación de señales y síntomas oculares.

\section{Las descripciones de las pruebas y procedimientos se presentan a continuación:}

Fijación visual - examinador posicionado detrás del niño, suspendiendo el aro con el borde inferior a la altura de su línea media, de 20 a $30 \mathrm{~cm}$ frente a sus ojos. El aro debe ser movido en giro y, a continuación, parado. La respuesta esperada es la fijación de la mirada hacia el aro durante, por lo menos, tres segundos.

Contacto ocular con el examinador / Sonrisa al contacto social - el examinador, situado frente al niño, debe inclinarse hasta que su cara esté alineada a 30 $\mathrm{cm}$ de la del niño. Debe sonreír y mover la cabeza de cuatro a cinco veces y vocalizar en dirección al niño en la prueba de la sonrisa al contacto social. La respuesta esperada es la mirada del niño hacia el examinador durante tres segundos (contacto ocular con el examinador) y la sonrisa o vocalización del primero (sonrisa al contacto social). 
Seguimiento visual horizontal / Seguimiento visual vertical - examinador posicionado detrás del niño, fuera de su campo de visión, suspendiendo el aro con el borde inferior a la altura de su línea media y de 20 a 30 $\mathrm{cm}$ frente a sus ojos. Con el niño mirando hacia el aro, este debe ser movido en sentido látero-lateral, en el seguimiento visual horizontal, y en el sentido céfalo-caudal en el seguimiento visual vertical, alternativamente. La respuesta esperada es que se produzca el seguimiento visual, por lo menos, una vez.

Exploración visual del ambiente - se observa el comportamiento visual exploratorio del niño cuando se sienta libre para seguir su propio interés en el ambiente de evaluación. La respuesta esperada es la exploración visual espontánea del ambiente.

Exploración visual de la mano - durante el examen, se observa el comportamiento del niño mientras se encuentra libre para seguir su propio interés. Esta prueba se hace durante períodos de reposo del niño, calmado y despierto, sin ningún estímulo. La respuesta esperada es mirar espontáneamente hacia las manos.

Aumento del movimiento de miembros superiores al visualizar el objeto - el examinador, enfrente del niño, debe suspender el aro por el cordón, hasta que la extremidad inferior esté en la línea media del niño, aproximadamente de 20 a $30 \mathrm{~cm}$ encima de sus ojos. El aro debe ser movido suavemente para atraer la mirada del niño y a continuación pararlo en la línea media. La respuesta esperada es aumento del movimiento de los miembros superiores mientras se mantenga contacto visual con el objeto.

Extender el brazo en la dirección del objeto visualizado - enfrente del niño, el examinador suspende el aro por el cordón hasta que la extremidad inferior esté en la línea media del niño, aproximadamente 20 a $30 \mathrm{~cm}$ encima de sus ojos. A continuación, mueve el aro suavemente para atraer la mirada del niño, y entonces lo mantiene parado en la línea media. La respuesta esperada es mover los miembros superiores a propósito en la dirección del aro mientras mira hacia él.

Los niños fueron examinados en un único momento e individualmente por el equipo de terapeutas ocupacionales entrenados, de los cuales uno fue el responsable de la aplicación y los registros de las pruebas, y los demás, de observar y registrar las respuestas en presencia de los padres o responsables del niño. Después de la aplicación del instrumento se realizaba comparación entre las respuestas registradas por el examinador y observadores.

También al final de cada examen se explicaba a los padres o responsables cómo habían respondido los niños al test. En el caso en que el niño no respondiese a alguna prueba, el equipo de investigación explicaba los posibles motivos para ese hecho, orientando a los padres para que estimulasen visualmente a sus hijos y continuasen en la asistencia terapéutica ocupacional.

Estos procedimientos fueron realizados en sala bien iluminada, climatizada y libre de cualquier estímulo sonoro, con una duración de 10 a 15 minutos. Durante la aplicación de las pruebas, los niños permanecían en posición de supino, sobre una superficie plana.

Participaron en este estudio 22 niños, dándose homogeneidad entre los sexos (50\% masculino y $50 \%$ femenino). Todos los niños fueron examinados una única vez y los resultados obtenidos nos muestran que $18(81,8 \%)$ presentan el diagnóstico de Síndrome de Down; los otros $4(18,2 \%)$ presentan otros síndromes o se encuentran con diagnóstico en estado de investigación. La media de edad cronológica de los bebés fue de 5,13 meses. Se observó que de los 22 niños examinados, el $86,3 \%$ está asistidos por el equipo multidisciplinar.

Cuadro 1. Guía de triaje para Examen de la Conducta Visual.

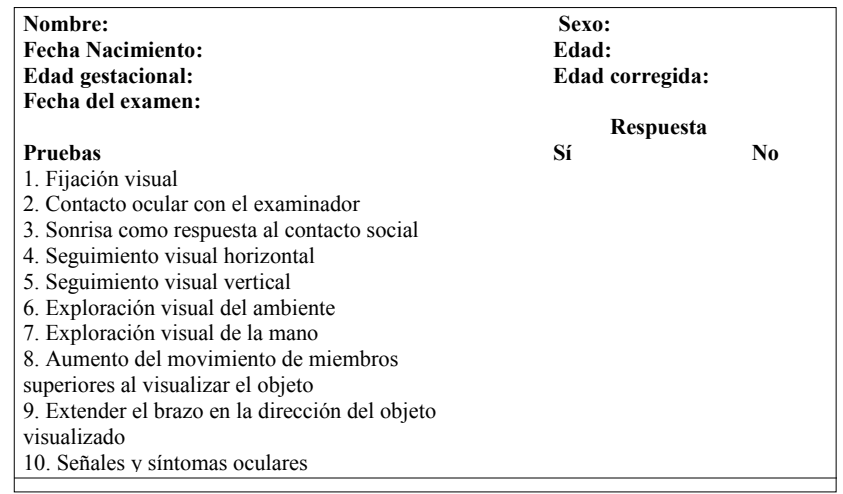

(Gagliardo, H. G. R. G. et al., 2004)

Este estudio presentó como criterio de inclusión niños de una franja de edad comprendida entre 15 días y 
12 meses, asistidos en la Asociación Novo Rumo, atendidos o no por la Terapia Ocupacional, y como criterio de exclusión, niños que presentasen ceguera total.

A los padres o responsables legales de los niños participantes les fueron explicados los objetivos y la metodología de la investigación, y la colecta de datos solo se inició después de la firma del término de consentimiento informado libre y voluntario).

Los datos fueron analizados y presentados en forma de frecuencia simple por medio del programa Microsoft Word Excel 2010.

El trabajo fue sometido al análisis del Comité de Ética para la Investigación con Seres Humanos de la Universidad Federal de Pernambuco (UFPE) mediante el CAAE n. 37037414.5.0000.5208, recibiendo infor- me favorable para su realización de acuerdo con el protocolo $n^{\circ} 897.669$.

\section{Resultados}

Con la aplicación de la Guía de Triaje para el examen visual, se observó que $63,6 \%$ de los niños no respondió a las pruebas de sonrisa al contacto social y extender el brazo en dirección al objeto visualizado, a pesar de que estos niños se encontraban en la franja de edad adecuada para una respuesta positiva, según lo que se observa en la tabla 1.

Tabla 1. Distribución de respuestas del comportamiento visomotor de 22 niños con alteración neurológica o genética. Asociación Novo Rumo, Recife, Pernambuco, 2015

\begin{tabular}{|c|c|c|c|c|}
\hline Pruebas & $\mathrm{N}$ & Sí (\%) & $\mathrm{N}$ & No (\%) \\
\hline Fijación visual & 21 & $95,4 \%$ & 1 & $4,5 \%$ \\
\hline Contacto ocular con el examinador & 20 & $90,9 \%$ & 2 & $9,0 \%$ \\
\hline Sonrisa como respuesta al contacto social & 8 & $36,3 \%$ & 14 & $63,6 \%$ \\
\hline Seguimiento visual horizontal & 19 & $86,3 \%$ & 3 & $13,6 \%$ \\
\hline Seguimiento visual vertical & 20 & $90,9 \%$ & 2 & $9,0 \%$ \\
\hline Exploración visual del ambiente & 18 & $81,8 \%$ & 4 & $18,1 \%$ \\
\hline Exploración visual de la mano & 12 & $54,6 \%$ & 10 & $45,4 \%$ \\
\hline Aumento del movimiento de miembros superiores & 15 & $68,1 \%$ & 7 & $31,8 \%$ \\
\hline Extender el brazo en la dirección del objeto visualizado & 8 & $36,3 \%$ & 14 & $63,6 \%$ \\
\hline Señales y síntomas oculares & 6 & $27,2 \%$ & 16 & $72,7 \%$ \\
\hline
\end{tabular}




\section{Discusión}

A pesar de que la población con síndrome de Down se ve más fácilmente afectada por alteraciones visuales debido a las mismas características da síndrome, fue posible constatar que los niños evaluados en este estudio presentaron un percentil elevado de respuestas positivas. Y esta respuesta puede estar relacionada con la estimulación que dichos niños reciben por el acompañamiento terapéutico prestado en la Institución de la que forman parte.

Moura et al (2008), Hassano (2011) y Ruas et al (2006), dicen que la sonrisa social comienza a aparecer alrededor del segundo o tercer mes de edad y que bebés con un mes de vida presentan aún un repertorio limitado de acciones, y en general todavía no manifiestan sonrisa social.

De acuerdo con Albuquerque et al (2013), la sonrisa social tiene la función de favorecer la socialización del niño y promover su desarrollo cognitivo. La ausencia de la sonrisa social también puede estar asociada a cuadros de aislamiento social, retrasos de lenguaje y dificultades sensoriales (Beline, Fernandes, 2007).

En el presente estudio, el 63,6\% de los niños examinados no respondió a la prueba de la sonrisa social. Al analizar este dato, es probable que los niños del estudio tengan poco contacto con personas que no sean de su familia, o poca estimulación de contacto con el otro, lo que puede interferir en su desarrollo psicosocial. Este dato direcciona la atención hacia los programas de follow-up, en vista de que la ausencia de estimulación en esta área podrá provocar posibles trastornos sociales, ya que la sonrisa constituye una relación social vital (Lima, et al., 2011).

La función apendicular de extender el brazo en dirección al objeto visualizado no fue respondida por la mayoría de los examinados. Este hecho puede estar relacionado con la hipotonía peculiar de los bebés con Síndrome de Down. De acuerdo con Ariani y Penasso (2005), la hipotonía muscular hace que el desarrollo inicial sea precario, y entonces, el niño tarda más en sonreír, agarrar objetos, balbucear, hablar, sujetar la cabeza, rodar, sentarse, arrastrarse, andar a gatas, andar y correr. De modo general, demuestran patrones atípicos para el control postural e incluso para la manipulación de objetos. Es importante destacar que la posición supina en que la prueba se desarrolla, los movimientos se producen contra la gravedad, exigiendo aún un mayor control de la coordinación muscular para aprender o alcanzar los objetos (Mattos, Bellani, 2010).

Ante los datos presentados en este estudio, vemos la necesidad de que profesionales de la salud y familiares de los niños despierten su atención hacia la importancia de la salud ocular para el desarrollo infantil, pues según Souza et al (2011), serán mayores las oportunidades de evitar alteraciones que puedan perjudicar el desarrollo pleno del niño.

Se tiene poco conocimiento de instrumentos de triaje simple que cualifiquen la visión de los niños, lo que convierte en un desafío el identificar instrumentos que puedan ser implantados en la rutina de servicios en forma de triaje visual, y que puedan ser utilizados por todos los profesionales terapeutas ocupacionales, en las prácticas de salud.

Formando parte del equipo multidisciplinar, el Terapeuta Ocupacional es un profesional habilitado para evaluar y estimular el desarrollo infantil, y por ello, debe estar atento a todos los sistemas relacionados en este proceso. En este sentido, el sistema visual también debe ser observado por este profesional, ya que las alteraciones visuales interfieren en el desarrollo global. Estas alteraciones pueden pasar desapercibidas por el profesional, y por eso, se habla de la necesidad de utilización de un método de triaje visual, por parte del terapeuta ocupacional en su intervención. Al poseer una evaluación visual, el terapeuta ocupacional puede, con su intervención, proporcionar una mejoría en el desempeño visual, viabilizar la interacción con el medio y la socialización, prevenir o minimizar desvíos en el comportamiento neuropsicomotor, cognitivo y social, promover una mayor independencia y autonomía, así como mejorar la calidad de vida de los individuos (Aragão et al. 2013; Montilha, Nobre \& Gagliardo, 2004).

La Terapia Ocupacional también tiene como objetivo la promoción de la salud. En este sentido, debe estar alerta para orientar a los padres sobre posibles alteraciones visuales, para que estos, en tiempo hábil, puedan buscar una orientación oftalmológica y obtener, así, un diagnóstico precoz. Además de proponer a los padres la búsqueda de un oftalmólogo, el terapeuta ocupacional podrá orientarlos para estimular la visión del niño. Silva y Cardoso (2009) confirman que la estimuحlación visual puede ser realizada por las propias madres en sus hogares, pues la enseñanza de actividades direccio $\neg$ nadas a la orientación materna acerca de los cuidados del niño podrá perfeccionar la asistencia prestada. 
El terapeuta ocupacional puede intervenir en el niño con déficit visual utilizando estrategias para suplir necesidades presentadas por el niño, como por ejemplo, la dificultad para localizar, discriminar y reconocer diferentes objetos. A través de su práctica utiliza recursos que contribuyen en el desarrollo del niño, de tal modo que este aprenderá a usar sus propios mecanismos para realizar y experimentar actividades para convertirse en un agente en su convivencia social (Mota, Marchiore \& Pinto, 2008). Con el conocimiento de dónde está localizado el déficit en el sistema visual, el terapeuta ocupacional es capaz de proyectar estrategias de evaluación y tratamiento adecuados para disminuir los problemas básicos y mejorar la función perceptual (Schneck, 2010).

De esta forma, la propuesta de la Guía de Evaluación de la Conducta Visual de Lactantes como rutina en la intervención del terapeuta ocupacional es pertinente, en vista de que se trata de un test simple, de fácil aplicación y de bajo coste, que califica el comportamiento visomotor.

\section{CONCLUSIONES}

Una vez expuesto que la guía de examen visual, tal como la utilizada en este estudio, nos ofrece respuestas importantes sobre el comportamiento visual de los niños, además de ser un procedimiento capaz de detectar posibles alteraciones visuales, queda demostrada la necesidad de su utilización en los servicios de salud.

La atención a la salud ocular no se debe producir tan solo cuando se identifican o diagnostican alteraciones visuales fácilmente perceptibles, como los desvíos oculares. El examen del comportamiento visomotor debe ser usado rutinariamente por tratarse de una medida de prevención y de intervención.

A pesar del número reducido de niños examinados en este estudio, se han presentado datos importantes para la evolución de la asistencia en la rehabilitación, considerando que esta investigación ha sido pionera en la institución donde el estudio se realizó.

Debe resaltarse que todos los niños deben pasar por exámenes de visión en los primeros meses de vida, principalmente aquellos considerados de riesgo (Sperandio, 1999). Este último constituye un motivo más para la inserción de la Guía en la práctica diaria, no solo en el lugar donde fue realizado este estudio, sino en los demás servicios de salud que prestan asistencia al niño.

Considerando el hecho de que el terapeuta ocupacional es el profesional capaz de evaluar el comportamiento visomotor de niños, este estudio refuerza la importancia que debe dársele al examen visual, además de fundamentar la práctica clínica de este profesional.

\section{ReFERENCIAS BibLIOGRÁfiCAS}

Aguiar, A. S. C., Cardoso, M. V. L. M. L. \& Lúcio, I. M. L. (2007). Teste do reflexo vermelho: forma de prevenção à cegueira. Revista Brasileira Enfermagem, 60 (5), p. 541-545. doi: 10.1590/S003471672007000500011

Albuquerque, R. C., Gagliardo, H. G. R. G. \& Barbosa, A. S. L. (2013). Expressão do sorriso social de lactentes pré-termo com idade corrigida. Revista Brasileira de Crescimento e Desenvolvimento Humano, 23 (3), p. 318-321.

Aragão, F. M., Vasconcelos, T. B., Silva, G. P. F., Montenegro, C. M., Câmara, T. M. S., Pires, J. L. V. R., Sousa, C. T., Macena, R. H. M., Bastos, V. P. D. (2013). A importância da estimulação visual em crianças com Síndrome de Down: visão de profissionais. Revista de Ciências Médicas e Biológicas, 12 (2), p. 207-213.

Ariani, C., Penasso, P. (2005). Análise clínica cinemática comparativa da marcha de uma criança normal e outra portadora de Síndrome de Down na fase escolar (7 a 10 anos). Reabilitar, 26 (7), p. 17-23.

Bayley, N. (1993). Bayley scales on infant development. 2.Ed, San Francisco: American Psychological Corporation.

Belini, A. E. G., Fernandes, F. D. M. (2007). Olhar de bebêsem desenvolvimento típico: Correções longitudinais encontradas. Revista da Sociedade Brasileira de Fonoaudiologia, 12 (3), p. 165-173.

Dargassies, S.A. (1997). Desarrollo neurológico del recién nacido de termino y prematuro. Buenos Aires: Medica Panamericana.

Ferreira, A. P. A. et al. (2011). Comportamento visual e desenvolvimento motor de recém-nascidos prematuros no primeiro mês de vida. Revista Brasileira de Crescimento e Desenvolvimento Humano, 21 (2), p. 335-343. 
Gagliardo, H. G. R. G. (2003). Contribuições de Terapia Ocupacional para detecção de alterações visuais na fonoaudiologia. Saúde em Revista, 5 (9), p. 89-94.

Gagliardo, H. G. R. G., Gonçalves, V. M. G. \& Lima, M. C. M. P. (2004), Método para avaliação da conduta visual de lactentes. Arquivos de Neuropsiquiatria, 62 (2), p. 300-306.

Gagliardo, H. G. R. G., Nobre, M. I. R. S. (2001) Intervenção precoce na criança com baixa visão. Revista Neurociências, 9 (1), p. 16-19.

Hassano, A. Y. S. (2011). Desenvolvimento Neuropsicomotor no Primeiro Ano de Vida. Revista de Pediatria SOPERJ, 12 (1), p. 9-14.

Khetpal, V., Donahue, S. P. (2010) Cortical visual impairment: Etiology, associated findings, andprognosis in a tertiarycare setting. American Journal of Occupational Therapy, 11 (3), p. 235-239.

Lima, A. C. D., et al. (2011). Comportamento visuomotor no primeiro e segundo mês de idades cronológica e corrigida de lactentes gemelares prematuros. Pediatria (São Paulo), 33 (3), p. 135-141.

Mattos, B. M., Bellani, C. D. F. (2010). A importância da estimulação precoce em bebês portadores de síndrome de Down: revisão de literatura. Revista Brasileira de Terapias e Saúde, 1 (1), p. 51-63.

Montilha, R. C. I., Nobre, M. I. R. S. \& Gagliardo, H. G. R. G. (2004). Atuação Terapêutico-ocupacional junto a pacientes com transtornos da visão. Em De Carlo, M. M. R. P. \& Luzo, M. C. M. (Ed. 1), Terapia Ocupacional: reabilitação física e contextos hospitalares (p. 276-291). São Paulo: Roca.

Motta, M. P., Marchiore, L. M. \& Pinto, J. H. (2008) Confecção de brinquedo adaptado: uma proposta de intervenção da terapia ocupacional com crianças de baixa visão. O Mundo da Saúde de São Paulo, 32 (2), p. 139-145.
Moura, M. L. S., Ribas, A. F. P., Seabra, K. C., Pessôa, L. F., Nogueira, S. E., Mendes, D. M. L. F., Rocha, S. B., Vicente, C. C. (2008). Interações mãe-bebê de um e cinco meses: aspectos afetivos, complexidade e sistemas parentais predominantes. Psicologia: Reflexão e Crítica, 21 (1), p. 66-73.

Pueschel, S. M. (2007). Características físicas da criança. En Pueschel, S. M. (Orgs.). Síndrome de Down: guia para pais e educadores (p. 77-83). Campinas: Papirus.

Ruas, T. C. B., Ravanini, S. G., Martinez, C. S., Gagliardo, H. G. R. G., Françoso, M. F. C., Rim, P. H. H. (2006). Avaliação do Comportamento Visual de Lactentes no primeiro e segundo meses de vida. Revista Brasileira de Crescimento e Desenvolvimento Humano, 16 (3), p. 1-8.

Schneck, C. M. (2010). Visual Perception. En Smith, C. \& Brien, O' (Ed. 6) Occupational Therapy for Children (p. 373-403). Missouri: MosbyElsevier.

Schwartzman, J. S. (2003). Generalidades. En Schwartzman, J. S. (Orgs). Síndrome de Down (p. 16-31). São Paulo: Memnon/Mackenzie.

Silva, G. R. F., Cardoso, M. V. L. M. L. (2009). Percepção de mães sobre um manual educativo sobre estimulação visual da criança. Revista Eletrônica de Enfermagem, 11 (4), p. 847-857.

Souza, N. C. M., et al. (2011). Comportamento visual e perfil socioeconômico e demográfico de recém-nascidos prematuros da Maternidade do Hospital das Clínicas de Pernambuco - UFPE. Arquivos Brasileiros de Oftalmologia, 74 (1), p. 33-36.

Sperandio, A. M. G. (1999). Promoção da saúde ocular e prevenção precoce de problemas visuais nos serviços de saúde pública. Revista de Saúde Pública, 33 (5), p. 513-520. 